\title{
Negative energy waves and quantum relativistic Buneman instabilities
}

\author{
F. Haas, ${ }^{1}$ B. Eliasson, ${ }^{2}$ and P. K. Shukla ${ }^{2,3}$ \\ ${ }^{1}$ Departamento de Física, Universidade Federal do Paraná, 81531-990 Curitiba, Paraná, Brazil \\ ${ }^{2}$ International Centre for Advanced Studies in Physical Sciences and Institute for Theoretical Physics, \\ Faculty of Physics \& Astronomy, Ruhr University Bochum, D-44780 Bochum, Germany \\ ${ }^{3}$ Department of Mechanical and Aerospace Engineering \& Center for Energy Research, University of California San Diego, \\ La Jolla, California 92093, USA
}

(Received 6 June 2012; published 18 September 2012)

\begin{abstract}
The quantum relativistic Buneman instability is investigated theoretically using a collective Klein-Gordon model for the electrons and a cold fluid model for the ions. The growth rate and unstable wave spectrum is investigated in different parameter regimes corresponding to various degrees of relativistic and quantum effects. The results may be important for streaming instabilities involving ion dynamics in very dense plasmas.
\end{abstract}

DOI: 10.1103/PhysRevE.86.036406

PACS number(s): 52.27.Ny, 52.35.Qz, 67.10.Hk

\section{INTRODUCTION}

The problem of the stability of electron beams propagating through a plasma is important in the context of laboratory beam-plasma experiments [1], inertial fusion schemes [2], in the solar corona [3], astrophysical objects [4,5], etc. It has been suggested that pulsar glitches are due to a streaming instability [6,7], where superfluid neutrons and superconducting protons co-exist with relativistic electrons [8]. It was early recognized that electron beams propagating through the plasma can give rise to high-frequency electron plasma waves, or low-frequency ion-acoustic waves. The ion motion becomes important if the electrons drift as a whole through the plasma and typically becomes unstable if the relative drift speed between the electrons and ions is larger than the ion acoustic speed, as in the original Buneman instability [9] in an unmagnetized plasma (or electrons streaming along the magnetic field lines) and the Farley-Buneman instability in magnetized plasmas [10].

The physics of the linear Buneman instability is fairly well understood [11] in the nonquantum $(\hbar=0)$ and nonrelativistic $(1 / c=0)$ cases ( $\hbar$ is Planck's constant divided by $2 \pi$ and $c$ is the speed of light in vacuum). In other situations, relatively thin beams of electrons penetrate a plasma consisting of both electrons and ions, giving rise to electron two-stream and ion Buneman instabilities in different parameter regimes in the nonrelativistic [12] and relativistic [13-15] regimes. An electron beam propagating through a plasma can give rise to electrostatic two-stream instabilities when the wave vector and electric field are aligned, and electromagnetic instabilities obliquely to the beam direction. A typical condition for the ion motion to become important in a current-neutral plasma is $\gamma \geqslant \alpha M /\left(m Z_{i}\right)$, with $\gamma$ being the electron beam $\gamma$ factor, $\alpha$ the beam to plasma density ratio, $Z_{i}$ the ion charge state, and $M$ and $m$ the ion and electron masses [12], respectively. To incorporate quantum effects, collective multistream Schrödinger [16] and Klein-Gordon [17] models have been used to investigate the electron quantum two-stream instability for nonrelativistic and relativistic cases. In the nonrelativistic regime, the quantum two-stream instability can be physically understood in terms of a free energy available due to negative energy modes [18]. In addition, low-frequency linear and nonlinear waves in collisional, nonrelativistic quantum plasmas can be derived using multistream, carrier-envelope methods [19].

In this paper, we consider the quantum relativistic Buneman instability, in which the bulk electrons stream against the ions, initially at rest. We compare the classical relativistic case with the nonrelativistic and quantum case, as well as with the combined quantum relativistic case. As a model, we use a collective Klein-Gordon model [20] for the electrons and a cold plasma model for the ions. The main advantage of the collective Klein-Gordon model over the Schrödinger-Poisson model is that it takes into account the relativistic effects, in addition to the quantum diffraction effects. For the sake of simplicity, we shall neglect the electron thermal and Landau damping effects, which can be included using a relativistic Wigner model [21].

The paper is organized in the following fashion. In Sec. II, the pertinent dielectric function and the dispersion relation are deduced, starting from the basic hydrodynamic equations for the two-species plasma. In Sec. III, assuming the low-frequency and a beam-plasma resonance condition, the corresponding Buneman instability is studied, in the case of negligible quantum effects. Moreover, the instability is intuitively understood in terms of positive and negative energy modes. Sections IV and V extend the treatment to the quantum nonrelativistic and quantum relativistic cases, respectively. Section VI discusses the validity conditions of the model, as well as the observability of the predictions found, for certain densities and beam speeds. Section VII contains the final remarks.

\section{DIELECTRIC FUNCTION}

The mathematical model is based on a collective KleinGordon equation for the electrons that is cast into a set of fluidlike equations, and a cold fluid model for the ions. The electrons are described by a Klein-Gordon field $\psi=R \exp (i S / \hbar)$, so that $R=R(x, t)$ and $S=S(x, t)$ can be viewed as, respectively, the amplitude and phase of the collective electron wave function. For simplicity, we consider electrostatic waves in $1+1$ dimensions, so that all quantities depend only on $(x, t)$. From the Klein-Gordon equation, the evolution equations for $R$ and $S$ are obtained 
as $[17,20]$

$$
\begin{aligned}
& R\left(\frac{1}{c^{2}} \frac{\partial^{2}}{\partial t^{2}}-\frac{\partial^{2}}{\partial x^{2}}\right) S-\frac{e R}{c^{2}} \frac{\partial \phi}{\partial t}+\frac{2}{c^{2}} \frac{\partial R}{\partial t}\left(\frac{\partial S}{\partial t}-e \phi\right) \\
&-2 \frac{\partial R}{\partial x} \frac{\partial S}{\partial x}=0 \\
& \frac{1}{c^{2}}\left(\frac{\partial S}{\partial t}-e \phi\right)^{2}-\left(\frac{\partial S}{\partial x}\right)^{2}-m^{2} c^{2}=\frac{\hbar^{2}}{R}\left(\frac{1}{c^{2}} \frac{\partial^{2}}{\partial t^{2}}-\frac{\partial^{2}}{\partial x^{2}}\right) R
\end{aligned}
$$

where $e$ is the magnitude of the electron charge. Equation (1) plays the role of a quantum relativistic electron continuity equation, while Eq. (2) is a relativistic Hamilton-Jacobi equation with a quantum Bohm-like $\propto \hbar^{2}$ correction term, for the electrons. The ion continuity and momentum equations for the ion fluid density $n_{i}$ and velocity $v_{i}$ are

$$
\begin{gathered}
\frac{\partial n_{i}}{\partial t}+\frac{\partial}{\partial x}\left(n_{i} u_{i}\right)=0, \\
\frac{\partial u_{i}}{\partial t}+u_{i} \frac{\partial u_{i}}{\partial x}=-\frac{e}{M} \frac{\partial \phi}{\partial x},
\end{gathered}
$$

respectively. Due to their larger mass, the ions are taken as nonrelativistic and nonquantum. Also, as a first approximation, no thermal effects are included for either the electrons and ions. The electrostatic potential $\phi$ is obtained from Poisson's equation,

$$
\frac{\partial^{2} \phi}{\partial x^{2}}=-\frac{e}{\varepsilon_{0}}\left[\frac{R^{2}}{m c^{2}}\left(\frac{\partial S}{\partial t}-e \phi\right)+n_{i}\right] .
$$

where $\varepsilon_{0}$ is the vacuum electric permittivity, in terms of the appropriate electron charge density, as discussed in more detail in Ref. [17].

Assuming that the electron fluid is streaming as a whole through the ionic fluid, we have an equilibrium solution,

$$
\begin{aligned}
& R=\sqrt{\frac{n_{0}}{\gamma}}, \quad S=-\gamma m c^{2} t+p x, \\
& n_{i}=n_{0}, \quad u_{i}=0, \quad \phi=0,
\end{aligned}
$$

in the reference frame where the ions are at rest, with the relativistic $\gamma$ factor $\gamma=\left[1+p^{2} /\left(m^{2} c^{2}\right)\right]^{1 / 2}$ together with $n_{0}$ as a the equilibrium density. Notice the modified equilibrium electron fluid density, due to the spatial contraction $(\gamma>1)$.

Linearizing the model equations around the equilibrium (6) with plane wave perturbations $\propto \exp [i(k x-\omega t)]$, we obtain the dielectric function,

$$
\begin{aligned}
\varepsilon= & 1-\frac{\omega_{i}^{2}}{\omega^{2}} \\
& -\frac{\omega_{e}^{2}}{\gamma} \frac{\left[1-\hbar^{2}\left(\omega^{2}-c^{2} k^{2}\right) /\left(4 m^{2} c^{4}\right)\right]}{\left[\gamma^{2}(\omega-k v)^{2}-\hbar^{2}\left(\omega^{2}-c^{2} k^{2}\right)^{2} /\left(4 m^{2} c^{4}\right)\right]},
\end{aligned}
$$

with the dispersion relation being $\varepsilon=0$. In Eq. (7), $\omega_{e}=$ $\left[n_{0} e^{2} /\left(m \varepsilon_{0}\right)\right]^{1 / 2}$ and $\omega_{i}=\left[n_{0} e^{2} /\left(M \varepsilon_{0}\right)\right]^{1 / 2}$ are the electron and ion plasma frequencies, respectively, and $v=p /(\gamma m)$ is the beam velocity.

It is instructive to consider the consequences of the dielectric function (7) for three separate cases: (a) relativistic nonquantum; (b) nonrelativistic quantum, and (c) joint relativistic and quantum.

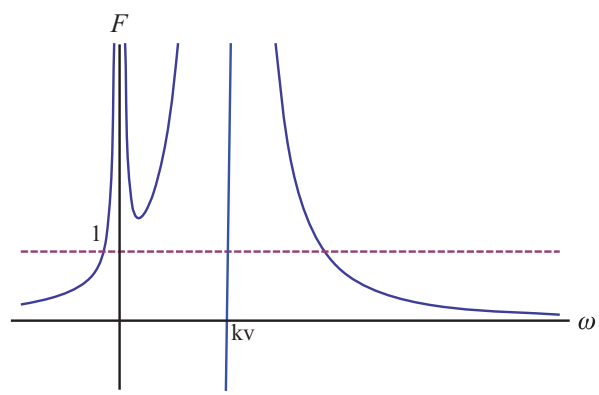

FIG. 1. (Color online) Characteristic function $F=F(\omega, k)$ from Eq. (8) in the nonquantum case, showing a generic unstable equilibrium when $F_{\min }>1$.

\section{RELATIVISTIC NONQUANTUM CASE}

Setting formally $\hbar=0$ in Eq. (7), we have

$$
\varepsilon=1-\frac{\omega_{i}^{2}}{\omega^{2}}-\frac{\omega_{e}^{2}}{\gamma^{3}(\omega-k v)^{2}}=1-F(\omega, k),
$$

which also defines a nonquantum characteristic function $F=$ $F(\omega, k)$. The dispersion relation $F=1$ is a fourth degree polynomial in the wave frequency $\omega$. From the graphics of the characteristic function, it follows that stability (four real solutions) is obtained when the minimum value $F_{\min }<1$. When $F_{\text {min }}>1$ one has two real and two complex conjugate roots, one of them an unstable mode. Finally, $F_{\min }=1$ is marginally stable. In Fig. 1 an unstable case is depicted.

Setting $\partial F / \partial \omega=0$ and solving for $\omega$, we obtain

$$
\omega_{\min }=\left(\frac{m}{M}\right)^{1 / 3} \gamma k v\left[1+\gamma\left(\frac{m}{M}\right)^{1 / 3}\right]^{-1},
$$

as the wave frequency for the minimum of the characteristic function. Correspondingly, we have

$$
F_{\min }=\frac{\omega_{e}^{2}}{\gamma^{3} k^{2} v^{2}}\left[1+\gamma\left(\frac{m}{M}\right)^{1 / 3}\right]^{3}>1,
$$

for instability. Hence, relativistic effects $(\gamma>1)$ tend to shrink the range of unstable wave numbers.

For the interpretation of the stability analysis, we first rewrite the dispersion relation as

$$
\left(\omega-\omega_{n}\right) \omega^{2}=\frac{\omega_{i}^{2}(\omega-k v)^{2}}{\omega-\omega_{p}},
$$

where

$$
\omega_{p}=k v+\frac{\omega_{e}}{\gamma^{3 / 2}}, \quad \omega_{n}=k v-\frac{\omega_{e}}{\gamma^{3 / 2}}
$$

are the relativistic version of the usual Doppler-shifted beam modes. Notice that when the ions are taken as immobile $(M \rightarrow$ $\infty)$, one has $\omega_{p, n}$ as exact normal modes.

It is interesting to focus on resonant wave numbers and low-frequency modes such that

$$
k v \simeq \frac{\omega_{e}}{\gamma^{3 / 2}}, \quad \omega \ll k v,
$$


implying that $\omega_{n} \simeq 0$. One then immediately obtains the estimates

$\omega-\omega_{p} \simeq-2 k v, \quad(\omega-k v)^{2} \simeq k^{2} v^{2}, \quad \omega-\omega_{n} \simeq \omega$,

so that Eq. (11) becomes

$$
\omega^{3}=-\frac{m}{2 M} \frac{\omega_{e}^{3}}{\gamma^{3 / 2}} .
$$

The reason why the dispersion relation (a fourth degree polynomial equation) can be converted into Eq. (15), which is of the third degree, is the assumption (13). Far from resonant or the high-frequency normal modes, one cannot detect this limiting case in this manner.

Equation (15) has one real root,

$$
\omega=-\left(\frac{m}{2 M}\right)^{1 / 3} \frac{\omega_{e}}{\sqrt{\gamma}},
$$

and two complex conjugate roots,

$$
\omega=\left(\frac{m}{16 M}\right)^{1 / 3} \frac{\omega_{e}}{\sqrt{\gamma}}(1 \pm i \sqrt{3}) .
$$

The plus sign in Eq. (17) corresponds to an exponentially growing mode. Note that the growth rate becomes smaller due to relativistic effects. In addition, for all three modes one obtains

$$
\frac{\omega}{k v} \sim \gamma\left(\frac{m}{M}\right)^{1 / 3},
$$

apart from numerical factors of order unity, so that the low-frequency assumption is acceptable with the exception of extremely relativistic beams (in the case of a hydrogen plasma where $m / M=1 / 1867$ ). Moreover, the instability condition (10) is also fulfilled by the resonant wave numbers in Eq. (13).

The time-averaged energy density $\langle W\rangle$ in a dielectric medium can be shown [22] to be given by

$$
\langle W\rangle=\frac{\varepsilon_{0}|\delta E|^{2}}{4} \frac{d}{d \omega}\left[\omega \varepsilon_{r}(\omega)\right]_{\omega_{r}},
$$

where $\delta E$ is the electric field perturbation and $\varepsilon_{r}, \omega_{r}$ are the real parts of the dielectric function and the wave frequency. In the present dissipation-free problem, we have $\varepsilon=\varepsilon_{r}$. In Eq. (19), both field and particle energy contributions are already taken into account.

From the above well-known result, it is found that the energy content of the different modes depends on the sign of the quantity,

$$
\omega \frac{\partial \varepsilon}{\partial \omega}=2 \frac{\omega_{i}^{2}}{\omega^{2}}+\frac{2 \omega_{e}^{2} \omega}{\gamma^{3}(\omega-k v)^{3}},
$$

calculated from Eq. (8) by considering $\omega=\omega_{r}$. By inspection, the ion contribution has always a positive energy. Concerning electrons, if the wave velocity is larger than the beam's mode the energy is positive. From Eq. (12) one has that $\omega=\omega_{p}$ is a positive energy mode, while $\omega=\omega_{n}$ is a negative energy mode (without loss of generality, we use in this work the convention $\omega>0, k>0)$. One can understand the result as follows. When the Doppler-shifted frequency of the beam mode is negative, its energy is also negative. Moving through the plasma, the

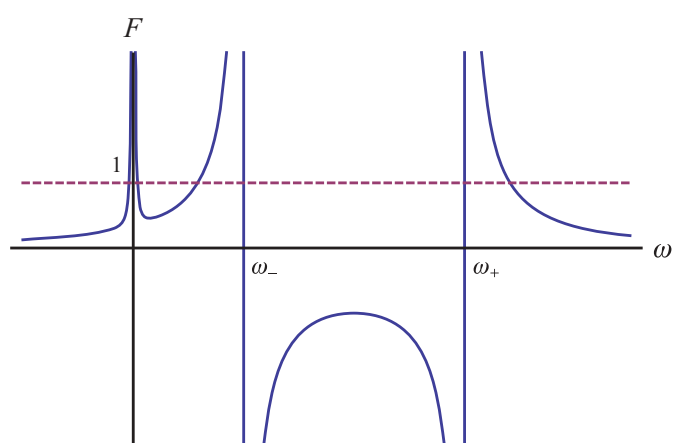

FIG. 2. (Color online) Characteristic function $F=F(\omega, k)$ from Eq. (21) in the nonrelativistic case, showing a generic stable equilibrium when $F_{\min }<1$. In the graphic, $\omega_{ \pm}=k v \pm \hbar k^{2} /(2 m)$.

beam is then slowed down, losing energy which is the seed for the growing amplitude of the wave.

Concluding the results in this section, we investigated the low-frequency instability due to a negative energy relativistic beam mode in an oscillating ionic background. Relativistic effects tend to produce a smaller range of unstable wave numbers, as well as a smaller growth rate, in comparison to the $\gamma \simeq 1$ case.

\section{NONRELATIVISTIC QUANTUM CASE}

Setting formally $1 / c=0$ in Eq. (7), we have

$$
\varepsilon=1-\frac{\omega_{i}^{2}}{\omega^{2}}-\frac{\omega_{e}^{2}}{(\omega-k v)^{2}-\hbar^{2} k^{4} /\left(4 m^{2}\right)}=1-F(\omega, k),
$$

which also defines a nonrelativistic characteristic function $F=F(\omega, k)$. The dispersion relation $F=1$ is again a fourth degree polynomial in the wave frequency $\omega$. However, there are qualitative changes in comparison with the nonquantum case, as apparent in Fig. 2 drawn for $v>\hbar k /(2 m)$. It can be verified that the maximum of the characteristic function for the frequencies $k v-\hbar k^{2} /(2 m)<\omega<k v+\hbar k^{2} /(2 m)$ is always negative. Hence one needs $F_{\min }>1$ for instability, where the corresponding wave frequency $\omega_{\min }$ satisfy $0<$ $\omega_{\min }<k v-\hbar k^{2} /(2 m)$. In passing, the case $v \leqslant \hbar k /(2 m)$ can be shown to produce only linearly stable oscillations, and will be not considered.

The dispersion relation $F=1$ can be rewritten as

$$
\left(\omega-\omega_{n}\right) \omega^{2}=\frac{\omega_{i}^{2}\left[(\omega-k v)^{2}-\hbar^{2} k^{4} /\left(4 m^{2}\right)\right]}{\omega-\omega_{p}},
$$

where

$$
\omega_{p}=k v+\left(\omega_{e}^{2}+\frac{\hbar^{2} k^{4}}{4 m^{2}}\right)^{1 / 2}, \quad \omega_{n}=k v-\left(\omega_{e}^{2}+\frac{\hbar^{2} k^{4}}{4 m^{2}}\right)^{1 / 2}
$$

are the quantum versions of the usual Doppler-shifted beam modes. Notice that when ions are taken as immobile $(M \rightarrow$ $\infty)$, one has $\omega_{p, n}$ as exact normal modes.

In an analogy with the previous section, it is interesting to focus on the wave numbers such that $\omega_{n} \simeq 0$ and on low 
frequencies, or

$$
k v \simeq\left(\omega_{e}^{2}+\frac{\hbar^{2} k^{4}}{4 m^{2}}\right)^{1 / 2}, \quad \omega \ll k v .
$$

Proceeding as before, the result is

$$
\omega^{3}=-\frac{m \omega_{e}^{3}}{2 M} \theta_{ \pm}(H),
$$

where

$$
H=\frac{\hbar \omega_{e}}{m v^{2}}
$$

measures the strength of quantum effects, and where it was defined that

$$
\theta_{ \pm}(H)=\frac{H}{\sqrt{2}}\left[1 \pm\left(1-H^{2}\right)^{1 / 2}\right]^{-1 / 2} .
$$

One should compare the relativistic and quantum expressions for the low-frequency beam modes in Eqs. (15) and (25), respectively. In addition, $H$ is bigger for dense plasmas, since $\omega_{e} \propto n_{0}^{1 / 2}$.

The resonance condition can be explicitly solved, yielding

$$
k v=\frac{\omega_{e}}{\theta_{ \pm}(H)},
$$

which gives real wave numbers provided that $H^{2} \leqslant 1$. In particular, the previous resonant wave number is now split in two, due to the quantum recoil term $\hbar^{2} k^{4} /\left(4 m^{2}\right)$ in Eq. (21). Moreover, one has

$$
\begin{aligned}
& \theta_{+}(H)=\frac{H}{2}\left(1+\frac{H^{2}}{8}\right)+O\left(H^{5}\right), \\
& \theta_{-}(H)=1-\frac{H^{2}}{8}+O\left(H^{4}\right),
\end{aligned}
$$

so that $\theta_{ \pm}$have, respectively, a purely quantum and a semiclassic nature. In Fig. 3 the behavior of $\theta_{ \pm}(H)$ is shown. The two modes coalesce when $H=1$.

Similar to the nonquantum case, Eq. (25) can be solved, yielding a purely oscillatory mode

$$
\omega=-\left(\frac{m \theta_{ \pm}(H)}{2 M}\right)^{1 / 3} \omega_{e},
$$

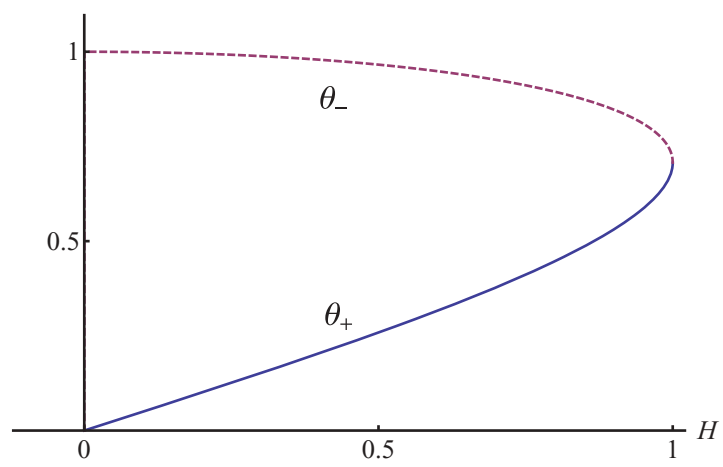

FIG. 3. (Color online) Functions $\theta_{ \pm}$from Eq. (27). Bottom, line: $\theta_{+}$. Upper, dashed: $\theta_{-}$. a damped mode

$$
\omega=\left(\frac{m \theta_{ \pm}(H)}{16 M}\right)^{1 / 3} \omega_{e}(1-i \sqrt{3}),
$$

and an exponentially growing mode

$$
\omega=\left(\frac{m \theta_{ \pm}(H)}{16 M}\right)^{1 / 3} \omega_{e}(1+i \sqrt{3}) .
$$

Now one has not only one, but two unstable low-frequency modes, according to the plus or minus sign chosen in $\theta_{ \pm}$. However, since $\theta_{ \pm}(H) \leqslant 1$, it is apparent from Eq. (32) that the quantum effects are stabilizing.

For consistency, it remains to check the low-frequency assumption. From Eqs. (30)-(32) it follows that

$$
\frac{\omega}{k v} \sim\left(\frac{m}{M}\right)^{1 / 3} \theta_{ \pm}^{4 / 3} \ll 1 .
$$

Besides, after some algebra one finds from Eq. (21)

$$
\begin{aligned}
\omega_{\min } & =\left[\frac{m}{M} \theta_{ \pm}(H)\right]^{1 / 3} \\
& \Rightarrow F_{\min } \simeq 1+3\left[\frac{m}{M \theta_{ \pm}^{2}(H)}\right]^{1 / 3}>1
\end{aligned}
$$

in accordance with the general instability condition.

Concerning the total energy, one should analyze the quantity

$$
\omega \frac{\partial \varepsilon}{\partial \omega}=\frac{2 \omega_{i}^{2}}{\omega^{2}}+\frac{2 \omega_{e}^{2} \omega(\omega-k v)}{\left[(\omega-k v)^{2}-\hbar^{2} k^{4} /\left(4 m^{2}\right)\right]^{2}} .
$$

By using Eq. (23) one concludes that $\omega=\omega_{p}$ and $\omega=\omega_{n}$ are positive and negative energy modes, respectively.

\section{RELATIVISTIC QUANTUM CASE}

Having in mind the fruitful results following from the lowfrequency assumption, in this work the full relativistic and quantum dispersion relation (7) will not be investigated in detail. Rather, it is interesting to restrict to slow waves such that

$$
\omega^{2} \ll c^{2} k^{2}
$$

which implies

$$
\varepsilon=1-\frac{\omega_{i}^{2}}{\omega^{2}}-\frac{\omega_{e}^{2}}{\gamma} \frac{\left[1+\hbar^{2} k^{2} /\left(4 m^{2} c^{2}\right)\right]}{\left[\gamma^{2}(\omega-k v)^{2}-\hbar^{2} k^{4} /\left(4 m^{2}\right)\right]} .
$$

The qualitative form of the characteristic function derived from Eq. (37) is similar to the nonrelativistic quantum characteristic function from Sec. IV, with quantitative changes due to $1 / c \neq$ $0, \gamma>1$.

Notice that fast wave propagation not satisfying Eq. (36) can be relevant in some instances, but is outside the scope of the present work. For example, the usual Bohm correction is not valid for waves with phase speed greater than the speed of light [23]. Moreover, the simplified dielectric function (37) does not produce the high-frequency pair modes [24], admitted by the fully relativistic dispersion relation. 
Once again, the dispersion relation $F=1$ is a fourth degree polynomial in the wave frequency $\omega$, which can be written as

$$
\left(\omega-\omega_{n}\right) \omega^{2}=\frac{\omega_{i}^{2}\left[(\omega-k v)^{2}-\hbar^{2} k^{4} /\left(4 \gamma^{2} m^{2}\right)\right]}{\omega-\omega_{p}},
$$

where

$$
\begin{aligned}
& \omega_{p}=k v+\left[\frac{\omega_{e}^{2}}{\gamma^{3}}\left(1+\frac{\hbar^{2} k^{2}}{4 m^{2} c^{2}}\right)+\frac{\hbar^{2} k^{4}}{4 \gamma^{2} m^{2}}\right]^{1 / 2}, \\
& \omega_{n}=k v-\left[\frac{\omega_{e}^{2}}{\gamma^{3}}\left(1+\frac{\hbar^{2} k^{2}}{4 m^{2} c^{2}}\right)+\frac{\hbar^{2} k^{4}}{4 \gamma^{2} m^{2}}\right]^{1 / 2} .
\end{aligned}
$$

Neglecting the ion correction, these are exact beam modes for the dielectric function (37). They correspond to Dopplershifted relativistic modifications of the usual Bohm-Pines dispersion relation [25].

Similarly to the nonquantum or nonrelativistic cases, it is useful to focus on the wave numbers such that $\omega_{n} \simeq 0$ and on low frequencies, or

$$
k v \simeq\left[\frac{\omega_{e}^{2}}{\gamma^{3}}\left(1+\frac{\hbar^{2} k^{2}}{4 m^{2} c^{2}}\right)+\frac{\hbar^{2} k^{4}}{4 \gamma^{2} m^{2}}\right]^{1 / 2}, \quad \omega \ll k v .
$$

The resonance condition gives

$$
\frac{k v}{\gamma}=\frac{\omega_{e}}{\varphi_{ \pm}(H, \gamma)},
$$

where

$$
\begin{aligned}
& \varphi_{ \pm}(H, \gamma) \\
& =\frac{H}{\sqrt{2}}\left\{1-\frac{\beta^{2} H^{2}}{4 \gamma^{3}} \pm\left[\left(1-\frac{\beta^{2} H^{2}}{4 \gamma^{3}}\right)^{2}-\frac{H^{2}}{\gamma^{5}}\right]^{1 / 2}\right\}^{-1 / 2},
\end{aligned}
$$

with $\beta=v / c$ and $H \neq 0$ given by Eq. (26).

Proceeding as before, we obtain the result

$$
\omega^{3}=-\frac{m \omega_{e}^{3}}{2 \gamma^{4} M} \varphi_{ \pm}(H, \gamma)\left(1+\frac{\beta^{2} \gamma^{2} H^{2}}{4 \varphi_{ \pm}^{2}(H, \gamma)}\right) .
$$

For $\gamma \rightarrow 1, \beta \rightarrow 0$, one has $\varphi_{ \pm}(H, \gamma) \rightarrow \theta_{ \pm}(H)$. Moreover, the $\propto \beta^{2}$ term in Eq. (44) vanishes, so that the quantum nonrelativistic results in Eqs. (25) and (28) are recovered. On the other hand, choosing $\varphi_{-}(H, \gamma)$ (the semiclassical branch) and then letting $H \rightarrow 0$ it is easy to verify that the relativistic, nonquantum results from Sec. III are recovered, considering $\varphi_{-}(H, \gamma)=\gamma^{5 / 2}+O\left(H^{2}\right)$.

It will be assumed that $\varphi_{ \pm}(H, \gamma)$ is not complex, which is true either for

$$
H<\frac{2 \gamma}{\beta^{2}}\left(\sqrt{\gamma}-\frac{1}{\sqrt{\gamma}}\right)=1+\beta^{2}+\frac{31 \beta^{4}}{32}+O\left(\beta^{6}\right)
$$

or for

$$
\begin{aligned}
H & >\frac{2 \gamma}{\beta^{2}}\left(\sqrt{\gamma}+\frac{1}{\sqrt{\gamma}}\right) \\
& =\frac{4}{\beta^{2}}+2+\frac{13 \beta^{2}}{8}+\frac{23 \beta^{4}}{16}+O\left(\beta^{6}\right) .
\end{aligned}
$$

Equation (45) extends the condition $H<1$ from the preceding section to the relativistic domain, while Eq. (46) seemingly

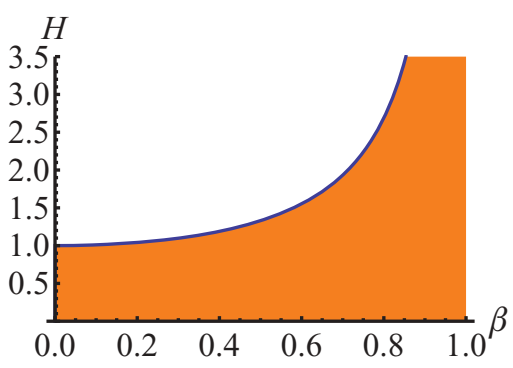

FIG. 4. (Color online) The filled area shows the allowable values of the quantum parameter $H$ in terms of $\beta=v / c$, as found from Eq. (45).

provides a new parameter regime not existent in the nonrelativistic approximation. However, in practice it can be verified that the inequality (46) can be attained only for nonrealistic values of $H$ (at least $H>10.8$ ). Hence, Eq. (45) gives the only important constraint, as shown in Fig. 4 below. We note that the relativistic effects allows bigger $H$ values.

From Eq. (44) it is obvious that the instability analysis from the preceding section applies, once the replacement $\theta_{ \pm}(H) \rightarrow$ $\zeta_{ \pm}(H, \gamma)$ is made, where

$$
\zeta_{ \pm}(H, \gamma) \equiv \frac{\varphi_{ \pm}(H, \gamma)}{\gamma^{4}}\left[1+\frac{\beta^{2} \gamma^{2} H^{2}}{4 \varphi_{ \pm}^{2}(H, \gamma)}\right] .
$$

Moreover, generalizing Eq. (29) one has

$$
\begin{aligned}
& \zeta_{+}(H, \gamma)=\frac{H}{2 \gamma^{2}}\left[1+\frac{\left(2-\gamma^{2}\right) H^{2}}{8 \gamma^{5}}\right]+O\left(H^{5}\right), \\
& \zeta_{-}(H, \gamma)=\frac{1}{\gamma^{3 / 2}}\left[1-\frac{\left(2-\gamma^{2}\right) H^{2}}{8 \gamma^{5}}\right]+O\left(H^{4}\right),
\end{aligned}
$$

so that $\zeta_{ \pm}(H, \gamma)$ have, respectively, a purely quantum and a semiclassic nature. In Fig. 5 the behavior of $\zeta_{ \pm}(H, \gamma)$ is shown for different values of the relativistic parameter $\gamma$. The two modes coalesce at the maximal quantum parameter found from Eq. (45). Overall, the relativistic effects produce a smaller value of $\zeta_{ \pm}(H, \gamma)$, and hence a smaller instability growth rate that is proportional to $\left[\zeta_{ \pm}(H, \gamma)\right]^{1 / 3}$. Finally, we note that $\omega=$ $\omega_{p}$ [Eq. (39)] can be shown to be a positive energy mode, while $\omega=\omega_{n}$ [Eq. (40)] is a negative energy mode.

\section{OBSERVABILITY ISSUES}

Here we discuss the validity of our model. We focus on the results of Sec. V, since they are the most general in the present investigation. In other words, the semirelativistic situation $\omega \ll c k$ is treated. We remark that the particles can still be relativistic, with beam velocities of the order of the speed of light. Considering the Buneman unstable modes and separating the real and imaginary parts according to $\omega=\omega_{R}+i \omega_{I}$, the conclusion was that

$$
\omega_{R}=\left(\frac{m \zeta_{ \pm}(H, \gamma)}{16 M}\right)^{1 / 3} \omega_{e},
$$

with $\omega_{I}=\sqrt{3} \omega_{R}$ and the wave numbers satisfying the resonance condition (42). The functions $\zeta_{ \pm}(H, \gamma)$ are defined in Eq. (47), which involves $\varphi_{ \pm}(H, \gamma)$, given by Eq. (43). The 

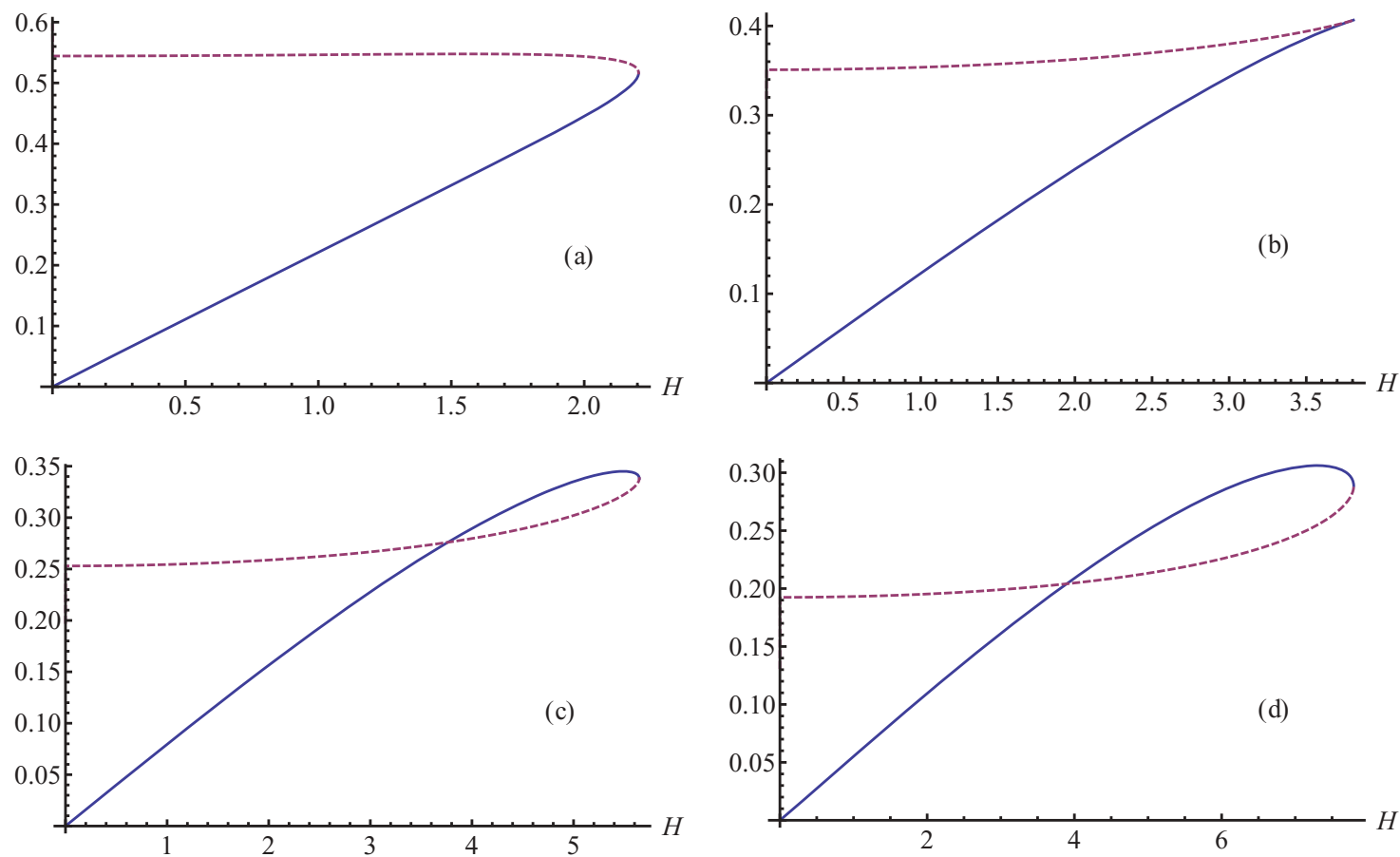

FIG. 5. (Color online) Functions $\zeta_{ \pm}(H, \gamma)$ from Eq. (47). In all plots, the dashed curve is $\zeta_{-}(H, \gamma)$, while $\zeta_{+}(H, \gamma)$ is the other one. Parameters are (a) upper left, $\gamma=1.5$; (b) upper right, $\gamma=2.0$; (c) bottom left, $\gamma=2.5$; (d) bottom right, $\gamma=3.0$.

basic parameters of the wave, therefore, are the density $n_{0}$ and the beam velocity $v$, from which $\omega_{R}$ and $k$ are obtained. Moreover, there are, in principle, two modes, corresponding to the positive or negative sign in $\zeta_{ \pm}, \varphi_{ \pm}$.

After numerically exploring the relevant parameter regimes allowed by the structural condition (45), which poses a limit on the density $n_{0}$ once the beam speed is chosen, it can be verified that the low-frequency condition $\omega_{R} \ll c k$ is always safely fulfilled. The same applies to the no-pair creation condition $\hbar \omega_{R} \ll 2 m c^{2}$, which is necessary since otherwise one would have to consider quantized fields in a QED formalism.

The Bohm-like term in the right hand side of the quantum relativistic Hamilton-Jacobi equation (2) follows directly from the Madelung transform of the Klein-Gordon equation $[17,20]$. Hence the domain of applicability is the same as for the Klein-Gordon equation, restricted to plasmas where the electron spin-1/2 effects are not decisive, like in most electrostatic phenomena. Moreover, in a multistream approach it is expected that each beam has a definite identity, which is satisfied if the thermal spread is not too large. In the nondegenerate case, this implies a beam velocity much larger than the thermal speed. In the degenerate case, the thermal speed is replaced by the Fermi speed. In a multistream model [26], one discretizes the (quasi)probability distribution function, representing it by a certain number $N$ of streams. In this way, one can, in principle, introduce thermal and kinetic effects like Landau damping, using a large number of streams. The result of a kinetic theory should then be recovered in the appropriate limit when $N \rightarrow \infty$. In this sense, any desired distribution function can be emulated by a sufficiently large number of streams. For instance, the Maxwell-Boltzmann or Fermi-Dirac equilibria can be modeled in this way.
Another fundamental issue is the applicability of the hydrodynamic method, which amounts to a small quantum recoil and associated resonances [23,26,27]. Examining the dielectric function (37), one finds that

$$
\gamma^{2}\left(\omega_{R}-k v\right)^{2} \gg \frac{\hbar^{2} k^{4}}{4 m^{2}}
$$

as a further requirement, posing a limit on the maximal wave number. Hence, as in any fluidlike theory, we have a long wavelength assumption.

One further question in the approach refers to the magnetic field generation due to the unbalanced current at equilibrium. Following the discussion of Bludman et al. [13], we can examine the neglect of static magnetic fields according to

$$
\nabla \times \mathbf{B}=-\mu_{0} n_{0} e \mathbf{v} \Rightarrow B \sim \mu_{0} n_{0} e L v,
$$

where $\mathbf{B}$ is the magnetic field strength, $\mu_{0}$ is the vacuum permeability, and $L$ is some characteristic dimension of a beam of finite cross section. From this, one can estimate the Larmor frequency $\omega_{L}$ as

$$
\omega_{L}=\frac{e B}{\gamma m} \sim \frac{n_{0} e^{2} \mu_{0} L v}{\gamma m}=\frac{\omega_{e}^{2} L v}{\gamma c^{2}} \Rightarrow \frac{\omega_{L}}{\omega_{e}}=\frac{\beta L \omega_{e}}{\gamma c} .
$$

For the low frequency, the last quantity may be significant, so that neglecting a return current may be unjustified. However, one can always have $\omega_{L} / \omega_{e} \ll 1$ for sufficiently small $L$.

Finally, no collisional effects are included. Therefore, a weak coupling assumption should hold. Since the form of the cold beam and weak coupling conditions depend on the degree of degeneracy, we treat separately the degenerate 
and nondegenerate cases, together with some representative examples. Moreover, the wavelength must certainly be (much) larger than the interparticle distance, otherwise clearly a fluidlike model would be not adequate.

\section{A. Degenerate case}

In the fully degenerate case, by definition one has $\kappa_{B} T \ll$ $E_{F}$, where $\kappa_{B}$ is the Boltzmann constant, $T$ is the beam temperature, and $E_{F}=\hbar^{2}\left(3 \pi^{2} n_{0}\right)^{2 / 3} /(2 m)$ the Fermi energy. The cold beam hypothesis then is equivalent to $v \gg v_{F}$, where $v_{F}=\left(2 E_{F} / m\right)^{1 / 2}$ is the Fermi speed.

On the other hand, the weak coupling condition amounts to $E_{p} \ll E_{F}$, since a measure of the kinetic energy of one charge carrier is the Fermi energy and where $E_{p}=e^{2} /\left(4 \pi \varepsilon_{0} a\right)$ is a measure of the electrostatic energy per particle. Here $a=$ $\left(4 \pi n_{0} / 3\right)^{-1 / 3}$ is the Wigner-Seitz radius.

Consider, for instance, a large density $n_{0}=10^{32} \mathrm{~m}^{-3}$ used in laser-compressed plasma interaction experiments $[28,29]$. To safely satisfy the cold beam assumption, one may chose $v=0.9 c$, so that $v_{F} / v=0.06$. One then finds that $T \ll 9.1 \times 10^{6} \mathrm{~K}$ is necessary for complete electron degeneracy. The ideality condition is also verified, since $E_{p} / E_{F}=0.14$.

Focusing on the negative sign choice in Eqs. (42) and (49), so that $\zeta_{-}(H, \gamma)$ and $\varphi_{-}(H, \gamma)$ are used, it is verified that the structural requirement (45) and the small quantum recoil inequality (50) are satisfied. Moreover, one has $\zeta_{-}^{1 / 3}=0.66$, which, by comparison with Eq. (49), means a reduction of $34 \%$ of the instability rate, due mostly to relativistic effects since $H=9.0 \times 10^{-4}$ with the chosen parameters. In addition, Eqs. (42) and (49) yield $\omega_{R}=12.1 \times 10^{15} \mathrm{~s}^{-1}$ and $k=$ $6.0 \times 10^{8} \mathrm{~m}^{-1}$, implying a wavelength $\lambda=2 \pi / k=10.5 \mathrm{~nm}$ much larger than the interparticle distance, since in this case $\lambda / a=782.7$.

Unfortunately, the choice of the positive sign in Eqs. (42) and (49), so that $\zeta_{+}(H, \gamma)$ and $\varphi_{+}(H, \gamma)$ are used, gives wavelengths much smaller than the interparticle distance $a$, in all reasonable parameter regimes. For instance, in the present example where $n_{0}=10^{32} \mathrm{~m}^{-3}, v=0.9 c$ one has $\lambda / a=0.04$ for the purely quantum wave. For such small wavelengths, a quantum relativistic kinetic theory is necessary. Therefore, more realistic predictions from the model can be trusted only when referring to the semiclassic mode (the negative sign choice). A further indication of the need of a kinetic theory consideration is the violation of the small recoil condition (50) for the purely quantum Buneman mode. Also, note that a more rigorous treatment of degenerate plasma would include exchange effects due to the particles indistinguishability.

\section{B. Nondegenerate case}

In the fully nondegenerate case, one has by definition $\kappa_{B} T \gg E_{F}$, and the cold beam hypothesis amounts to $v \gg v_{T}$, where $v_{T}=\left(3 \kappa_{B} T / m\right)^{1 / 2}$ is the thermal velocity. On the other hand, the weak coupling condition amounts to $E_{p} \ll \kappa_{B} T$.

Consider, as a representative example, a density $n_{0}=$ $10^{24} \mathrm{~m}^{-3}$ and a beam velocity $v=0.9 c$. The simultaneous weak coupling and cold beam hypothesis then imply that
$2.7 \times 10^{3} \mathrm{~K} \ll T \ll 1.6 \times 10^{9} \mathrm{~K}$. The nondegeneracy condition is immediately satisfied, since the Fermi temperature $T_{F}=E_{F} / \kappa_{B}=42.3 \mathrm{~K}$ in this case. Moreover, focusing on the negative sign choice in Eqs. (42) and (49), so that $\zeta_{-}(H, \gamma)$ and $\varphi_{-}(H, \gamma)$ are used, it is verified that the structural requirement (45) and the small quantum recoil inequality (50) are satisfied. One further has $\zeta_{-}^{1 / 3}=0.60$, which, by comparison with Eq. (49), means a reduction of $40 \%$ of the instability rate, due mostly to relativistic effects, since $H=$ $9.0 \times 10^{-8}$ with the chosen parameters. In addition, Eqs. (42) and (49) yield $\omega_{R}=1.1 \times 10^{12} \mathrm{~s}^{-1}$ and $k=8.0 \times 10^{4} \mathrm{~m}^{-1}$, implying a wavelength $\lambda=7.9 \times 10^{-5} \mathrm{~m}$ much larger than the interparticle distance, since in this case $\lambda / a=1.3 \times 10^{4}$. The same comments as earlier on the purely quantum mode apply, since using $\zeta_{+}$and $\varphi_{+}$would produce $\lambda / a=9.5 \times$ $10^{-5}$, which deserves a kinetic theory treatment. This fact is further justified by the violation of the inequality (50) also in the nondegenerate case, when choosing the new quantum mode.

\section{SUMMARY AND CONCLUSIONS}

In summary, we have developed a unified methodology for the treatment of low-frequency beam-driven instabilities in relativistic quantum plasmas. A detailed comparison was made considering different parameter regimes (formally, $\hbar=0$ and $1 / c \neq 0 ; \hbar \neq 0$ and $1 / c=0$; both $\hbar \neq 0$ and $1 / c \neq 0)$. The corresponding Buneman instabilities were shown to be associated to certain beam-plasma negative energy modes. Both quantum and relativistic effects cause a stabilizing effect, with the remark that the quantum effects produce a splitting of the original Buneman mode into two modes, due to the quantum recoil term as discussed in Sec. IV. In addition, observe that the model equations have certain limitations in particular because of the semiclassic nature (no quantized fields); see [17] for more details. Moreover, our collective Klein-Gordon plasma model neglects collisions, thermal and degeneracy pressure, and kinetic (Landau damping) effects, besides exchange interactions [26], which should be included in more sophisticated models for relativistic beams in quantum plasmas. Nevertheless, the dispersion relation (7) can be equally found using more complete, field-theoretic methods [24]. In this context, the positive-negative energy waves found here have a more broad applicability, as long as the low-frequency assumption is valid.

As a by-product, the present treatment derived the Doppler-shifted relativistic Bohm-Pines dispersion relations in Eqs. (39) and (40). The relativistic effects inhibit dispersion, with a lower group velocity for a given wavelength, as can be verified. This prediction should be tested, e.g., with the development of multi-Peta-Watt lasers [30].

Regarding the observability considerations of Sec. VI, we note that while some restrictions on the practical aspects of the new Buneman unstable quantum branch were found, it does not mean that the mode should be ruled out. Rather, since it violates both the small recoil and long wavelength conditions, it certainly deserves a quantum relativistic kinetic theory. Nevertheless, the present hydrodynamiclike treatment indicates the existence of a qualitatively new mode, originating from quantum diffraction effects causing the split of the 
resonance condition (41) in two distinct solutions. It is an interesting issue, to investigate this new physical phenomenon in the context of relativistic kinetic theory containing quantum diffraction effects. However, this is outside the scope of the present work.

\section{ACKNOWLEDGMENTS}

This work was supported by Conselho Nacional de Desenvolvimento Científico e Tecnológico (CNPq), as well as by the Deutsche Forschungsgemeinschaft through Project No. SH21/3-2 of the Research Unit 1048.
[1] L. E. Thode and R. N. Sudan, Phys. Rev. Lett. 30, 732 (1973); Phys. Fluids 18, 1552 (1975).

[2] M. Tabak, J. Hammer, M. E. Glinsky, W. L. Kruer, S. C. Wilks, J. Woodworth, E. M. Campbell, M. D. Perry and R. J. Mason, Phys. Plasmas 1, 1626 (1994); M. Tabak, D. S. Clark, S. P. Hatchett, M. H. Key, B. F. Lasinski, R. A. Snavely, S. C. Wilks, R. P. J. Town, R. Stephens, E. M. Campbell, R. Kodama, K. Mima, K. A. Tanaka, S. Atzeni, and R. Freeman, ibid. 12, 057305 (2005).

[3] A. Klassen, M. Karlicky, and G. Mann, Astron. Astrophys. 410, 307 (2003).

[4] J. A. Zensus, Astron. Astrophys. 35, 607 (1997).

[5] T. Piran, Rev. Mod. Phys. 76, 1143 (2004).

[6] N. Andersson, G. L. Comer, and R. Prix, Phys. Rev. Lett. 90, 091101 (2003).

[7] N. Andersson, G. L. Comer, and R. Prix, Mon. Not. R. Astron. Soc. 354, 101 (2004).

[8] L. Samuelsson, C. S. Lopez-Monsalvo, N. Andersson, and G. L. Comer, Gen. Relativ. Gravit. 42, 413 (2010).

[9] O. Buneman, Phys. Rev. Lett. 1, 8 (1958).

[10] D. T. Farley, J. Geophys. Res. 68, 6083 (1963); O. Buneman, Phys. Rev. Lett. 10, 285 (1963).

[11] R. J. Briggs, in Advances in Plasma Physics, edited by A. Simon and W. B. Thompson, Vol. 4 (John Wiley and Sons, New York, 1971), p. 43.

[12] A. Bret, Phys. Scr. 84, 065507 (2011).

[13] S. A. Bludman, K. M. Watson, and M. N. Rosenbluth, Phys. Fluids 3, 747 (1960).
[14] A. Bret and M. E. Dieckmann, Phys. Plasmas 15, 012104 (2008).

[15] A. Bret, M. E. Dieckmann, and L. Gremillet, Ann. Geophys. 28, 2127 (2010).

[16] F. Haas, G. Manfredi, and M. R. Feix, Phys. Rev. E 62, 2763 (2000).

[17] F. Haas, B. Eliasson, and P. K. Shukla, Phys. Rev. E 85, 056411 (2012).

[18] F. Haas, A. Bret, and P. K. Shukla, Phys. Rev. E 80, 066407 (2009)

[19] F. Haas and A. Bret, Europhys. Lett. 97, 26001 (2012).

[20] T. Takabayasi, Prog. Theor. Phys. 9, 187 (1953).

[21] J. T. Mendonça, Phys. Plasmas 18, 062101 (2011).

[22] L. D. Landau and E. M. Lifshitz, Electrodynamics of Continuous Media (Pergamon, Oxford, 1960).

[23] D. B. Melrose and A. Mushtaq, Phys. Plasmas 16, 094508 (2009).

[24] V. Kowalenko, N. E. Frankel, and K. C. Hines, Phys. Rep. 126, 109 (1985).

[25] D. Bohm and D. Pines, Phys. Rev. 92, 609 (1953).

[26] S. V. Vladimirov and Yu. O. Tsyshetskiy, Phys. Usp. 54, 1243 (2011).

[27] P. K. Shukla and B. Eliasson, Phys. Usp. 53, 51 (2010); Rev. Mod. Phys. 83, 885 (2011).

[28] H. Azechi et al., Laser Part. Beams 9, 193 (1991).

[29] R. Kodama et al., Nature (London) 412, 798 (2001).

[30] G. A. Mourou, T. Tajima, and S. V. Bulanov, Rev. Mod. Phys. 78, 309 (2006); M. Marklund and P. K. Shukla, ibid. 78, 591 (2006). 